Benjamin SCHINDLER, St. Gallen ${ }^{1}$

\title{
Die föderale Kompetenzverteilung in der Schweizerischen Eidgenossenschaft aus historischer Sicht
}

\section{A Historical Perspective on the Distribution of Powers in the Swiss Confederation}

The distribution of powers in the Swiss Confederation has a long tradition dating back to the time before 1798 but was also influenced by American constitutional law. Today's attribution of competences has, however, departed considerably from its original model and largely resembles a functional division of tasks, with the legislative and governmental powers situated at the federal level and the administrative and judicial powers resting with the cantons.

Keywords: sovereignty - Swiss constitutional law - Swiss federalism

\section{Einleitung}

Warum Maria Theresia bis heute einen Schweizer Gerichtssaal ziert

Laufenburg ist ein schmuckes mittelalterliches Städtchen mit einem im Jahr 1525 errichteten Gerichtsgebäude. Darin findet sich ein prächtiger Verhandlungssaal mit unverändert erhaltener Ausstattung aus der Zeit des Spätbarocks (1770/1771). An den Wänden prangen nicht nur ein bekrönter Doppeladler mit dem Wappen Österreichs (Rot-Weiß-Rot) und Laufenburgs (Habsburger Löwe), sondern auch drei prunkvolle Herrscherbilder Maria Theresias, Franz' I. und Josephs II. Ein typisch österreichischer Gerichtssaal - so möchte man meinen. Doch unter den Augen ihrer apostolischen Majestäten urteilen heute Bezirksrichter des Schweizer Kantons Aargau, denn im Jahr 1797 endete mit dem

\footnotetext{
${ }^{1}$ Mein besonderer Dank geht an meine Mitarbeiterin Elena Dittli (B.A. HSG in Law) für ihre wertvollen Recherchen und an Dr. phil. Linus Hüsser für seine unverzichtbaren Hinweise zur Geschichte Vorderösterreichs.
}

Einmarsch französischer Truppen die seit 1173 bestehende Herrschaft der Habsburger in Laufenburg. Nach einem Intermezzo als französisches Protektorat und Distriktshauptort des helvetischen Kantons Fricktal wurde der linksrheinische Teil des Städtchens 1803 dem neu geschaffenen Kanton Aargau einverleibt.

Dass der Gerichtssaal 250 Jahre wie eine Zeitkapsel überdauert hat, mag in Anbetracht der militärischen Besetzung durch Frankreich, dem Wechsel zur Schweiz und den verfassungsrechtlichen Zäsuren des 19. Jahrhunderts erstaunen. Er steht aber für eine erstaunliche Kontinuität staatlicher Herrschaft auf dem Gebiet der heutigen Schweiz seit der Frühen Neuzeit. In Laufenburg urteilten noch im jungen Kanton Aargau Richter, die ihre Ausbildung an österreichischen Universitäten genossen hatten. Neben dieser personellen Kontinuität fand auch das österreichische Recht vorerst weiter Anwendung, denn bis zur Kodifikation des aargauischen Rechts galten in den zum Kanton Aargau zusammengefügten Gebieten die bisherigen Rechtsordnungen weiter. Und schließlich wurde das neu geschaffene Aargauer Recht dank einflussreicher 
Laufenburger Juristen nachhaltig von österreichischen Kodifikationsvorlagen geprägt. Dies gilt vor allem für das Strafrecht, das Zivilrecht, das Schulrecht und die Übernahme der staatlichen Gebäudeversicherung. Die Helvetisierung Laufenburgs war somit im Rechtsalltag nicht als radikaler Bruch spürbar, sondern gestaltete sich eher als evolutionärer Prozess gegenseitiger Annäherung, der auch zu einer teilweisen Austriakisierung des aargauischen Rechts führte.

Eine vergleichbare evolutionäre Entwicklung liegt auch der föderalen Kompetenzordnung im Schweizer Staatswesen zugrunde. Um diese Ordnung zu verstehen, genügt es daher nicht, mit der Darstellung dieser Geschichte erst im Jahr 1848 - dem Jahr der ersten Bundesverfassung - einzusetzen. Denn so wie das aargauische Justizwesen bis heute auf Fundamenten früherer Jahrhunderte gründet, wurden auch für die erste Bundesverfassung Bausteine aus früheren Epochen verwendet.

\section{Alte Eidgenossenschaft bis $\mathbf{1 7 9 8}$}

\section{"Von der eigentlichen Constitution [...] dieser Eydgenössischen Republic"}

Das Gebiet der heutigen Schweiz entsprach bis 1798 in weiten Teilen - ausgenommen die Gebiete wie dem bereits erwähnten vorderösterreichischen Fricktal oder Teilen des heutigen Kantons Genf - der Alten Eidgenossenschaft. Diese lässt sich aus rechtlicher Perspektive am ehesten mit einem komplexen Netzwerk unterschiedlicher Bündnisse zwischen ungleichen Partnern beschreiben. ${ }^{2}$ Eingebunden waren neben den vollberechtigten „Orten“ (Kantonen) auch „Zugewandte Orte" mit minderem Rechtsstatus. Zum Gebiet gehörten sodann Untertanengebie-

\footnotetext{
2 Exemplarisch IsELIN, Tentamen 11: „Corpus Helveticum est systema compositum ex multis civitatibus, quae autonomiam variis temporibus variis titulis adeptae, in societatem paulatim coierunt [...].".
}

te, die als "Gemeine Herrschaften" teilweise gemeinsam verwaltet wurden. Das institutionelle Forum dieses Bündnisgeflechts bildete die „Tagsatzung“ als Gesandtenkongress der einzelnen "Orte“. In den heutigen Lehrbüchern zum Schweizer Verfassungsrecht wird dieses Gebilde meist als Staatenbund bezeichnet; 3 gemeint ist damit eine Verbindung gleichberechtigter und souveräner Einzelstaaten auf Grundlage eines völkerrechtlichen Vertrags. Die Idealkategorien von „Staatenbund“ und „Bundesstaat" sind allerdings zu vereinfachend, um die Alte Eidgenossenschaft adäquat zu erfassen. Die Ungleichheit der Beteiligten und die unterschiedlichen Grade der Zugehörigkeit entziehen sich einer Systematisierung nach heutigen dogmatischen Kategorien. Unklar ist sodann, ob die rechtlichen Grundlagen der Eidgenossenschaft nach heutigem wie damaligem Verständnis als Verträge oder in ihrer Gesamtheit als Verfassung zu qualifizieren waren. Zweifelsohne lag diesem Gebilde keine formelle Verfassungsurkunde zugrunde. Zahlreiche der geschlossenen Bündnisse galten indes auf "ewig“ und sahen keine Kündigungsmöglichkeit vor. Es erstaunt daher nicht, wenn in der zeitgenössischen Literatur von "leges fundamentales" ${ }^{\text {" oder einer }}$ „eigentlichen Constitution [...] dieser Eydgenössischen Republic ${ }^{\prime \prime}$ die Rede war. Diese Verfassung kannte zudem bereits Ansätze einer föderalen Kompetenzausscheidung. Beschrieben wurde diese von Franz Michael Büeler (16421725) in seinem 1696 verfassten „Compendium des gemeinen eidgenössischen Rechts". Darin unterschied er das "gemein Eydtgnossische Recht" oder "Jus Publicum Helvetiae", das in allen Orten der Eidgenossenschaft Geltung bean-

\footnotetext{
${ }^{3}$ HÄFELIN u.a., Bundesstaatsrecht, Rz. 35f.; RHINOW, SCHEFER, UEBERSAX, Verfassungsrecht, Rz. 571f.; TSCHANNEN, Staatsrecht, Rz. 67, 243.

${ }^{4}$ BÜELER, Compendium 51 (fälschlich als 9 paginiert); vgl. auch WÜRGLER, Verfassungsrecht 52.

${ }^{5}$ Hans Jacob LEU, Vorrede zu SIMLER, Loblichen Eydgenossenschaft V.
} 
spruchte und vom Recht der einzelnen Orte geschieden war. ${ }^{6}$ Durch den Geltungsanspruch des gemeineidgenössischen Rechts wurde die Souveränität der einzelnen Orte „in etwelchen Sachen etwas limitiert undt eingeschränket ${ }^{\prime \prime}{ }^{7}$. Hier klingt bereits die Vorstellung einer geteilten Souveränität im Bundesstaat an, wie sie Alexis de Tocqueville (1805-1859) später für die USA artikulieren sollte. ${ }^{8}$ Büeler versuchte schließlich, die verschiedenen Bereiche des "Jus Publicum Helvetiae" thematisch zu gruppieren und beschrieb fünf Sachbereiche gemeineidgenössischen Rechts: Interne Streitbeilegung und Landesverteidigung, Förderung des Freihandels, Eindämmung von Zollhindernissen, Verwaltung der "Gemeinen Herrschaften“ und Bekämpfung von Missbräuchen im Münzwesen. Hier wurde also bereits das Bemühen deutlich, unter thematischen Gesichtspunkten verschiedene Zuständigkeitssphären voneinander abzugrenzen. In einzelnen Sachbereichen entschied die Tagsatzung sodann nicht einstimmig, sondern anerkannte auch Mehrheitsentscheidungen. ${ }^{9}$ Dies galt besonders bei der Verwaltung der "Gemeinen Herrschaften“, wo sich erste Ansätze gemeinsamer Staatlichkeit am deutlichsten zeigten. ${ }^{10}$ In einer Gesamtbetrachtung ließe sich mit der Gewaltenteilungskonzeption von John Locke (1632-1704) argumentieren, dass Exekutive und Legislative weitgehend in den Händen der einzelnen „Orte“ blieben, während die Föderative (,the power of war and peace, leagues and

\footnotetext{
${ }^{6}$ BÜELER, Compendium 50 (fälschlich als 8 paginiert).

${ }^{7}$ BÜELER, Compendium 52 (fälschlich als 10 paginiert). Vgl. auch IsELIN, Tentamen 23.

${ }^{8}$ Toceueville, Vol. I, 183.

${ }^{9}$ ISELIN, Tentamen 24: „ibi enim pars minor maiorem semper sequitur“; WÜRGLER, Tagsatzung 570.

${ }^{10}$ Ikonographisch kam dies in einem gemeinsamen, aus den Wappen der einzelnen „Orte“ zusammengesetzten Wappenschild zum Ausdruck, das von einer gemeinsamen Souveränitätskrone überwölbt wurde, MAISSEN, Republic 295.
}

alliances“11) dem kollektiv gebildeten Ganzen zustand. ${ }^{12}$

\section{Mediationsakte von 1803 "constituée fédérale par la nature"}

Nach dem Einmarsch französischer Truppen im Jahr 1798 brachen die Staatsstrukturen der Alten Eidgenossenschaft zusammen. Das „Ancien Régime“" wich der "Helvetischen Republik", deren Verfassung vom 12. April 1798 weitgehend eine Adaption der französischen Direktorialverfassung von 1795 darstellte. Artikel 1 der Verfassung bezeichnete die Helvetische Republik nach französischem Vorbild als "eins und unteilbar" („La République helvétique est une et indivisible.“). Im Unterschied zum französischen Original wurde diese Bestimmung aber um einen Absatz ergänzt, der den Bruch mit der Vergangenheit deutlich unterstrich. ${ }^{13}$ Die Helvetische Republik erwies sich trotz dieser verfassungsrechtlichen Rhetorik als äußerst uneins und instabil, und die Gegensätze zwischen Unitariern und Föderalisten spitzten sich immer mehr zu. Der Erste Konsul Frankreichs - Napoléon Bonaparte (1769-1821) - versuchte mit der von ihm auf seinem Landsitz Malmaison diktierten Verfassung vom 29. Mai 1801, eine föderal geprägte Republik zu verwirklichen, indem die Kompetenzen der Zentralgewalt und der Kantone im zweiten

\footnotetext{
${ }^{11}$ LOCKE, Two Treatises, Second Treatise, Chapter XII, Sect. 146.

12 MAISSEN, Republic 572.

13 „Es giebt keine Grenzen mehr zwischen den Cantonen und den unterworfenen Landen noch zwischen einem Canton und dem andern. Die Einheit des Vaterlandes und des allgemeinen Interesse's vertritt künftig das schwache Band, welches verschiedenartige, außer Verhältnis ungleich große, und kleinlichen Localitäten oder einheimischen Vorurtheilen unterworfene Theile zusammenhielt und auf Gerathewohl leitete. Man verspürte nur die ganze Schwache einzelner Theile; man wird aber durch die vereinigte Starke Aller stark sein." (ASHR 1, 567).
} 
Abschnitt der Verfassung je einzeln aufgezählt wurden. ${ }^{14}$ Diese „Verfassung von Malmaison“ scheiterte in der Folge aber am Widerstand der Unitarier. Nachdem die Helvetische Republik zusehends in kriegsähnlichen Wirren versank, berief Bonaparte die Konfliktparteien im September 1802 zu einer "Consulta“ nach Paris ein. Obwohl die Unitarier dort in der Mehrheit waren, stellte der Erste Konsul klar, dass nur eine föderale Struktur die Konflikte nachhaltig befrieden könne. Seiner Ansicht nach musste die Schweiz von Natur aus föderal verfasst sein („,constituée fédérale par la nature“). ${ }^{15}$ Das Ergebnis war die "Mediationsakte“ vom 19. Februar 1803, die in neunzehn Kapiteln die Kantonsverfassungen enthielt und im 20. Kapitel die „Bundesakte“. Die 1798 untergegangen "Orte“ erwachten nun als Kantone wieder zu neuem Leben, wobei aus den ehemals beherrschten Untertanengebieten, den nicht vollberechtigten "Zugewandten Orten" und den neu hinzu gekommenen Gebieten - wie dem Fricktal - neue und gleichberechtigte Kantone entstanden. Einer davon war der Aargau. Um die Gleichheit der Kantone zu unterstreichen, wurden sie in der Mediationsakte in alphabetischer Reihenfolge aufgeführt. Die Bundesakte wies dem Bund vergleichsweise wenige Kompetenzen zu, etwa zur Sicherstellung des freien Warenverkehrs, zur Vereinheitlichung des Münzwesens sowie die Bereiche der Außen- und Verteidigungspolitik. In Art. 12 verankerte die Bundesakte sodann eine subsidiäre Generalkompetenz der Kantone: „Die Kantone üben alle Gewalt aus, die nicht ausdrücklich der Bundesbehörde übertragen ist.“ (im französischen Original: „Les cantons jouissent de tous les pouvoirs qui n'ont pas été

\footnotetext{
14 ASHR 6, 933f.

15 So die von Bonaparte unterzeichnete Präambel der Mediationsakte vom 19.2. 1803; die französischsprachige Originalakte ist unter Wikimedia Commons frei zugänglich.
}

expressément délégués à l'autorité fédérale. ${ }^{“ 16}$ ) Die Bestimmung erinnert stark an Art. II der amerikanischen Konföderationsartikel von $1777^{17}$ und das 1791 verabschiedete zehnte Amendment zur Unionsverfassung von $1787 . .^{18}$ Die Ähnlichkeit dürfte kein Zufall sein, ${ }^{19}$ war doch einer der Konferenzleiter und möglichen Redaktoren der Bundesurkunde der französische Senator Jean-Nicolas Démeunier (17511814) - ein profunder Kenner des amerikanischen Verfassungsrechts wie auch der Schweizer Verhältnisse. ${ }^{20}$

Nach dem Ende der napoleonischen Vorherrschaft dominierten auch in der Schweiz restaurative Tendenzen und die Mediationsakte wurde abgelöst durch den Bundesvertrag (BV) vom 7. August 1815. Der Vertrag betonte zwar die Souveränität der Kantone, führte aber mit Ausnahme des Münzwesens die in der Bundesakte von 1803 bereits genannten „Angelegenheiten des Bundes“ weiter (§ 8 BV 1815). Die Gleichheit der Kantone wurde trotz restaurativen Tendenzen nicht mehr angetastet und die Abschaffung der „Unterthanenlande“ sogar ausdrücklich erwähnt ( 7 BV 1815). Auch wenn die Rechtsgrundlage des Vertrags auf einen Staatenbund hindeutete, ${ }^{21}$ konnte die Tagsatzung im Bereich der Bundesangelegenheiten mit einfachem oder qualifiziertem Mehr entscheiden (§ 8 BV 1815).

\footnotetext{
${ }^{16}$ Mediationsakte 103.

17 ,Each state retains its sovereignty, freedom and independence, and every Power, Jurisdiction and right, which is not by this confederation expressly delegated to the United States, in Congress assembled."

18 „The powers not delegated to the United States by the Constitution, nor prohibited by it to the States, are reserved to the States respectively, or to the people." ${ }^{19} \mathrm{Vgl}$. auch BLUMER, MOREL, Handbuch 209; BURCKHARDT, Kommentar 13; HIS, Staatsrecht 1, 100f.; RÜTTIMANN, Bundesstaatsrecht 28, 54f.

${ }^{20}$ Vgl. MONNIER, KÖLZ, Bonaparte 12f., 72ff.

${ }^{21}$ So die einhellige Einschätzung der heutigen Staatsrechtslehre: HÄFELIN u.a., Bundesstaatsrecht Rz. 42; RHINOW, SCHEFER, UEBERSAX, Verfassungsrecht, Rz. 572; TsCHANNEN, Staatsrecht, Rz. 67, 243.
} 
Dass dieses Gebilde kein idealtypischer Staatenbund war, wurde auch daran ersichtlich, dass die Tagsatzung wiederholt selbst die Bezeichnung „Bundesstaat“22 verwendete und in der Literatur dem Bundesvertrag Elemente einer Verfassung attestiert wurden ${ }^{23}$ oder der Vertrag schlicht als „Bundesverfassung“24 bezeichnet wurde.

\section{Bundesverfassung von 1848 Verwendung von "vielen und vortrefflichen Bausteinen"}

\section{a) Grundsatz: Subsidiäre}

\section{Generalkompetenz der Kantone}

Nach 1830 wurden die restaurativen Kräfte in zahlreichen Kantonen durch eine liberale „Regenerations"-Bewegung verdrängt. Elf Kantone gaben sich eine neue Kantonsverfassung und 1832 beschloss die Tagsatzung, auch den Bundesvertrag zu erneuern. Es wurde eine „Revisionscommission" eingesetzt, die am 15. Dezember 1832 den Entwurf für eine „Bundeskurkunde" vorlegte, der von der Tagsatzung beraten und in angepasster Form am 15. Mai 1833 verabschiedet wurde. Das erklärte Ziel des ursprünglichen Entwurfs wäre es gewesen, die Schweiz im ersten Artikel zu einem „unauflöslichen Bundesstaat" zu erklären - eine Formulierung, die der Tagsatzung zu gewagt schien, weshalb sie in der Fassung von 1833 fallen gelassen wurde. Beide Entwürfe sahen indes in Art. 2 Abs. 1 eine gleichlautende Bestimmung zur Kompetenzverteilung zwischen Bund und Kantonen vor: „Die Kantone sind souverän und üben als solche alle Rechte aus, die nicht ausdrücklich der Bundesgewalt übertragen sind." Diese Entwürfe basierten auf Art. 12 der Bundesakte von 1803, griffen mit der ausdrücklichen Erwähnung der Kanto-

\footnotetext{
22 Vgl. für die Jahre 1815 und 1816 KAISER, Amtliche Protokolle 1816, 824, 840.

${ }^{23}$ DuBS, Das Oeffentliche Recht 38.

${ }^{24}$ BLunTSCHLI, Geschichte 482.
}

nalsouveränität aber auch ein Element des Bundesvertrags von 1815 auf. Trotz der durch die Tagsatzung vorgenommenen Anpassungen scheiterte der Entwurf von 1833 an der Ratifizierung durch die Kantone - die Bevölkerung des für die katholische Zentralschweiz wichtigsten Kantons Luzern hatte sich dagegen ausgesprochen. Die Reformbestrebungen der 1830er-Jahre erwiesen sich im Nachhinein betrachtet dennoch als wichtiger Schritt. Denn die Entwurfstexte bildeten die Basis, auf deren Grundlage die Bundesverfassung von 1848 entworfen wurde. Nachdem die liberalen Kräfte im raschen und vergleichsweise unblutigen Sonderbundskrieg von 1847 über ein Separatbündnis katholischkonservativer Kantone gesiegt hatten, setzte die Tagsatzung im August 1847 erneut eine Revisionskommission ein, die innert kürzester Zeit (zwischen dem 17. Februar und dem 8. April 1848) den Text für eine Bundesverfassung vorlegte. Der Präsident der Kommission - der Berner Vertreter an der Tagsatzung und später einer der ersten Bundesräte Ulrich Ochsenbein (1811-1890) - formulierte den Bauplan für die neue Bundesverfassung so: „Am alten Gebäude sind viele und vortreffliche Bausteine, darunter alle Bestimmungen, welche von Abtretungen einzelner Souveränitätsrechte an den Bund handeln." 25 So wurde aus den Entwürfen von 1832 und 1833 schließlich Art. 3 der Bundesverfassung von 1848: „Die Kantone sind souverän, soweit ihre Souveränität nicht durch die Bundesverfassung beschränkt ist, und üben als solche alle Rechte aus, welche nicht der Bundesgewalt übertragen sind." Die Gründe für die Weglassung des Wortes „,ausdrücklich“ lassen sich - soweit ersichtlich - nicht mehr eruieren. ${ }^{26}$ Die Be-

${ }^{25}$ Zit.n. dem Protokoll von Friedrich Frey-Herosé, HOLENSTEIN, Stunde Null 490.

${ }^{26}$ BluMER, MOREL, Handbuch 210; BURCKHARDT, Kommentar 13. Während die bis heute wohl herrschende Lehre dieser Weglassung keine grundlegende Bedeutung zumisst und auf das Prinzip der Einzelermächtigung verweist (vgl. schon FLEINER, Bundes- 
stimmung wurde später nahezu unverändert zu Art. 3 der totalrevidierten Bundesverfassungen von 1874 und 1999. Sieht man von der Anrufung Gottes ab, dann ist dies die einzige Norm in der Schweizer Bundesverfassung, die seit 1848 nie geändert wurde.

Art. 3 der Bundesverfassung bringt zum Ausdruck, dass die Verfassungsväter von 1848 der Überzeugung waren, dass die Ursprünge staatlicher Herrschaft bei den Kantonen liegen - daher verfügen die Kantone über eine „vermute$\mathrm{te}^{{ }^{\prime \prime 27}}$ subsidiäre Generalkompetenz, und neue Bundeskompetenzen können nur durch ausdrückliche Einzelermächtigung der Verfassung geschaffen werden (Verfassungsvorbehalt zu Lasten des Bundes). ${ }^{28}$ Dass Art. 3 ausdrücklich nur die Souveränität der Kantone erwähnte,

staatsrecht 43; DuBS, Das Oeffentliche Recht 188; RÜTTIMANN, Bundesstaatsrecht 55), sahen BLUMER, MOREL (Handbuch 209f.) und SCHOLLENBERGER (Kommentar, 100f., 103f.) darin noch eine bewusste Weglassung.

${ }^{27}$ Diese Vermutung bezieht sich nur auf die Frage, ob eine Bundeskompetenz besteht. Einmal begründete Bundeskompetenzen sollen hingegen nach den etablierten Auslegungsmethoden ausgelegt werden und nicht restriktiv zu Gunsten der Kantone: BIAGGINI, Kommentar, Art. 3 Rz. 8; BURCKHARDT, Kommentar 14; HäFELIN u.a., Bundesstaatsrecht Rz. 1075; RHINOW, SCHEFER, UEBERSAX, Verfassungsrecht Rz. 718; noch anderer Meinung FleINER, GIACOMETTI, Bundesstaatsrecht 75; SCHOLLENBERGER, Kommentar 106-108.

${ }^{28} \mathrm{Vgl}$. den Bericht über den Entwurf einer Bundesverfassung vom 8.4.1848, erstattet von der am 16.8. 1847 von der Tagsatzung ernannten Revisionskommission (Bern 1848) 11: „Wir haben also vereinigte Staaten oder wenn man will einen Bundesstaat, ein System, das wesentlich vom Unitarismus verschieden ist; weil unsere Verbindung von Staaten eine Conföderation bildet, in welchen die Kantone souverän bleiben." Vgl. auch das Votum von Josef Munzinger (1791-1855), Vertreter des Kantons Solothurn in der Verfassungskommission und später einer der ersten Bundesräte: „Wollen wir geben oder wollen wir nehmen? Geben können wir nicht, was wir nie hatten, also muss man nehmen. Nehmen von der Souveränität der Kantone, aber in bescheidenem Maße und nur, in soweit es zum Glück des Ganzen nöthig ist", zit.n. HOLENSTEIN, Stunde Null 488. nicht aber diejenige des Bundes (etwa im Bereich der Außenpolitik), war kaum Zufall.29 Auch sonst fällt auf, dass die Bundesverfassung von 1848 eine staatenbündische Tonalität pflegte. Art. 1 zählte die „Völkerschaften der zwei und zwanzig souveränen Kantone" auf - bemerkenswerterweise nicht in alphabetischer Reihenfolge wie noch die Bundesakte von 1803, sondern in derjenigen des Bundesvertrags von 1815. Damit wurde subtil in Erinnerung gerufen, dass vor 1798 einige Kantone gleicher waren als andere. Der Begriff „Bundesstaat“ wurde erneut gemieden, es war nur vom „Bund“ oder der „Bundesgewalt“ die Rede. Offizielle Bezeichnung des neuen Staatswesens wurde der schon lange übliche deutschsprachige Begriff "Schweizerische Eidgenossenschaft" sowie in den lateinischen Sprachen die mit dem Bundesvertrag von 1815 etablierten Begriffe "Confédération suisse" bzw. "Confederazione Svizzera", die eher auf eine Konföderation und damit einen Staatenbund hindeuteten. Man kann diese Rhetorik mit bloßen „Bequemlichkeitsrücksichten“30 erklären oder darin einen geschickten Schachzug sehen, die im Sonderbundskrieg unterlegenen Föderalisten zu versöhnen. ${ }^{31}$

Die staatenbündische Rhetorik kontrastierte freilich mit dem Umstand, dass die neue Verfassung das Mehrheitsprinzip zur Grundregel machte und den Bund zu einer vollständigen Staatsgewalt ausbaute. Die Tagsatzung lebte zwar in gewisser Weise mit dem Ständerat weiter, in dem jeder Kanton mit zwei Mitgliedern und die „Halbkantone“ mit je einem Mitglied vertreten waren. Dessen Mitglieder waren aber ausdrücklich nicht mehr an Instruktionen gebunden (Art. 79 BV 1848) und somit höchstens noch politisch betrachtet "Vertreter" ihrer Kantone. Nach dem Vorbild des US-amerikanischen

${ }^{29}$ BLUNTSCHLI, Geschichte 514 (,Die Souveränetät des Bundes ist mit Absicht nicht ausdrücklich erwähnt“.).

${ }^{30}$ DuBS, Das Oeffentliche Recht 187.

${ }^{31}$ Vgl. BLUNTSCHLI, Geschichte 514. 
Repräsentantenhauses trat neben den Ständerat sodann der Nationalrat als Vertretung der schweizerischen Gesamtbevölkerung. Beide Kammern entschieden mit einfacher Mehrheit (Art. 77 BV 1848). Sodann genügte zur Annah$\mathrm{me}^{32}$ und Änderung der Bundesverfassung die einfache Mehrheit des (Gesamt-)Volkes und die Mehrheit der Kantone („Ständemehr“; Art. 114 BV 1848). Während bislang die Mehrheit über die Ausübung bereits bestehender Bundeskompetenzen entscheiden konnte, war neu auch die Mehrheit für die Begründung neuer Bundeskompetenzen ausschlaggebend. Auch wenn Art. 3 der Bundesverfassung rein sprachlich Kontinuität ausdrückte, erhielt die Bestimmung durch die deutlich erleichterte Möglichkeit zur Begründung neuer Bundeskompetenzen eine ganz andere Dynamik. Diese Dynamik konnte sich indes erst nach der Totalrevision von 1874 entfalten, als mit dem konzeptionellen Wandel zum Vollzugsföderalismus eine politischemotional entscheidende Hürde auf dem Weg zur Begründung neuer Bundeskompetenzen beseitigt worden war.

\section{b) Konkrete Ausgestaltung: Konsolidierung und sanfter Ausbau der Kompetenzen}

Welche konkreten Kompetenzen wies nun die Verfassung von 1848 dem Bund zu? Auch hier wird das Bemühen um Kontinuität sichtbar, indem die Verfassung in erster Linie bereits bestehende Bundeskompetenzen bestätigte, diese maßvoll erweiterte oder Bereiche zur Bundessache erklärte, die bereits gemäss bisheriger Praxis als Bundesaufgabe betrachtet wurden oder aufgrund interkantonaler Vereinbarungen („Konkordate") weitgehend vereinheitlicht waren.

\footnotetext{
${ }^{32}$ Art. 1 der Übergangsbestimmung blieb diesbezüglich unbestimmt. Die Tagsatzung stellte in ihrem Beschluss über die Annahme der Bundesverfassung vom 12. 9. 1848 auf die Mehrheit der Kantone und der Bevölkerung ab.
}

Sanft erweitert wurden die Bundeskompetenzen in den Bereichen Außen- und Außenhandelspolitik (Art. 8 BV 1848). ${ }^{33}$ Stärker ausgeweitet wurden die bestehenden Kompetenzen des Bundes in den Bereichen Zölle (Art. 22-28 BV 1848)34 und freier Warenverkehr (Art. 29-32 BV 1848). ${ }^{35}$ Die Befugnis zur Vereinheitlichung von Maß und Gewicht wurde dem Bund zugewiesen, wobei die Kompetenznorm ausdrücklich auf die "Grundlage des bestehenden eidgenössischen Konkordates" verwies (Art. 39 BV 1848). ${ }^{36}$ Auch weitere Kompetenzen, etwa zur Errichtung öffentlicher Werke (Art. 21 BV 1848), ${ }^{37}$ zur Regelung der Heimatlosen-Frage (Art. 56 BV 1848) (38 $^{38}$ sowie betreffend Fremdenpolizei (Art. 57 BV 1848) $)^{39}$ und Seuchenpolizei (Art. 59 BV 1848) $)^{40}$ erklärten Bereiche zu Bundeszuständigkeiten, die aufgrund von Tagsatzungsbeschlüssen oder Konkordaten bereits schweizweit geregelt waren.

Schließlich wurden aber auch neue Kompetenzen des Bundes begründet. Dem Bund wurde

\footnotetext{
${ }^{33}$ Vgl. bereits Art. 31-33 Bundesakte 1803 und §8 Abs. 3, 4 und 5 BV 1815; zur Änderung im Einzelnen BURCKHARDT, Kommentar 79-81; KLEY, Bundeskompetenzen 192.

${ }^{34} \mathrm{Vgl}$. bereits Art. 5, 6 Bundesakte 1803 und $\S 3$ und 11 BV 1815; KLEY, Bundeskompetenzen 193; zur Änderung im Einzelnen BuRCKHARDT, Kommentar 208211; KLEY, Bundeskompetenzen 193.

35 Vgl. bereits Art. 5 Bundesakte 1803 und § 11 BV 1815; zur Änderung im Einzelnen BuRCKHARDT, Kommentar 222-224.

${ }^{36}$ Vgl. ebd. 338f.

${ }^{37}$ Die Tagsatzung hatte 1783 einen Auftrag zur Korrektion des Flusses Linth erteilt, der allerdings erst $1804 \mathrm{zu}$ konkreten Bauplänen führte und zwischen 1807 und 1823 ausgeführt wurde. Die Tagsatzung fühlte sich für dieses "Schweizer Nationalwerk" zuständig, obwohl ihr weder vor 1798 noch aufgrund der Mediationsakte von 1803 oder dem BV 1815 eine ausdrückliche Kompetenz zugewiesen worden wäre; vgl. BURCKHARDT, Kommentar 154.

${ }^{38} \mathrm{Zu}$ den vorbestehenden Konkordaten ebd. 607f.

${ }^{39} \mathrm{Zu}$ den Beschlüssen der Tagsatzung und den Konkordaten ebd. 506, 628.

${ }^{40} \mathrm{Zu}$ den Beschlüssen der Tagsatzung und den Konkordaten ebd. $611 \mathrm{f}$.
} 
die Befugnis eingeräumt, „eine Universität und eine polytechnische Schule zu errichten“ (Art. 22 BV 1848), ${ }^{41}$ er sollte das Postwesen übernehmen (Art. 33 f. BV 1848) 42 $^{2}$ und die „Oberaufsicht über die Straßen und Brücken, an deren Erhaltung die Eidgenossenschaft ein Interesse hat" wahrnehmen (Art. 35 BV 1848). ${ }^{43}$ Und schließlich sollten dem Bund das Münzregal (Art. 36 BV) ${ }^{44}$ und das Pulverregal (Art. 38 BV 1848) ${ }^{45}$ zukommen. Der Bund machte von seinen Kompetenzen unterschiedlich rasch Gebrauch: Vordringlich erschien die Übernahme des chaotischen Postwesens durch den Bund, das dieser noch Ende 1848 an die Hand nahm, ${ }^{46}$ im Sommer 1849 trat die eidgenössische Zollgesetzgebung in $\mathrm{Kraft}^{47}$ und zwischen 1850 und 1852 wurde der Franken als Schweizer Münzwährung eingeführt. ${ }^{48} 1855$ schließlich nahm die Eidgenössisch Polytechnische Schule in Zürich ihren Betrieb auf. ${ }^{49}$

Dort, wo der Bund in bestehende Kompetenzen der Kantone eindrang oder diese verdrängte, wurde auf einen nahtlosen Übergang geachtet. Regelungslücken wurden auch dort vermieden, wo bestehende Tagsatzungsbeschlüsse und Konkordate von neuen Regeln des Bundesgesetzgebers abgelöst wurden. ${ }^{50}$ Die Bundeskompetenzen wirkten somit allesamt "nachträglich derogierend" oder "konkurrierend“. ${ }^{51}$ Auch

${ }^{41}$ Zur Vorgeschichte ebd. $195 f$.

${ }^{42}$ Zur Vorgeschichte ebd. 306f.; KLEY, Bundeskompetenzen $192 \mathrm{f}$.

${ }^{43}$ Eine analoge Bestimmung enthielt bereits Art. 23 der Bundesakte von 1803, nicht mehr aber der BV 1815, vgl. BURCKHARDT, Kommentar 315f.

${ }^{44}$ Zur Vorgeschichte ebd. 324f.; KLEY, Bundeskompetenzen 193f.

${ }^{45}$ Zur Vorgeschichte BURCKHARDT, Kommentar 342.

${ }^{46}$ KLEY, Bundeskompetenzen 192f.

${ }^{47}$ Ebd. 193.

${ }^{48}$ Ebd. 193f.

${ }^{49} \mathrm{Vgl}$. BURCKHARDT, Kommentar 199.

${ }^{50}$ Vgl. Art. 6 Übergangsbestimmung BV 1848; KLEY, Bundeskompetenzen 190.

${ }^{51}$ Zum Begriffsverständnis EHRENZELLER, EHRENZELLER, Kompetenzzuteilung 56f.; HäFELIN u.a., Bundesstaatsrecht Rz. 1092; RHINOW, SCHEFER, UEBERSAX, Ver- wenn gewisse der neu begründeten Bundeskompetenzen in der Schweizer Lehre seit Mitte des 20. Jahrhunderts in Anlehnung an die deutsche Dogmatik als „ursprünglich derogierend“ oder "ausschließlich“ qualifiziert werden, ${ }^{52}$ ist diese Zuordnung historisch gesehen unzutreffend. ${ }^{53}$

Die Bundesverfassung von 1848 markierte staatsorganisatorisch einen Umbruch und Neuanfang, indem mit dem Bund eine zusätzliche und vollwertige Staatsebene geschaffen wurde und diese mit gewaltengliedrig organisierten Staatsorganen - einem zweikammerigen Parlament (Bundesversammlung mit National- und Ständerat), einer Regierung (Bundesrat) und einem (vorerst noch schwachen) Bundesgericht ausgestattet wurde. Aus dieser Perspektive erscheint das Jahr 1848 als „Stunde Null“ - oder man kann in der Bundesverfassung gar eine „Neuerfindung der Schweiz" erblicken. ${ }^{54}$ Betrachtet man indes die Verteilung von Zuständigkeiten zwischen Bund und Kantonen, dann trifft diese Einschätzung nur bedingt zu..$^{55}$ Sowohl in der Grundsatzfrage wie auch in der konkreten Ausgestaltung ist die Verfassung von 1848 neben Neuerungen auch stark von Kontinuität und Rücksichtnahme gegenüber den Kantonen geprägt. Die Diskrepanz zwischen der neuartigen Staatsarchitektur und dem vergleichsweise bescheidenen Kompetenzzuwachs fiel auch dem in Heidelberg lehrenden Schweizer Juristen Johann Caspar Bluntschli (18081881) auf: „Das Missverhältnis zwischen der Großartigkeit des Organismus und der engen

fassungsrecht Rz.727f.; TsCHANNEN, Staatsrecht, Rz. 763.

52 Fleiner, Giacometti, Bundesstaatsrecht 102. In dieser Tradition HÄFELIN u.a., Bundesstaatsrecht Rz. 1098; RHINOW, SCHEFER, UEBERSAX, Verfassungsrecht Rz. 725f.; TSCHANNEN, Staatsrecht, Rz. 765.

${ }^{53}$ BURCKHARDT, Kommentar 16; KLEY, Bundeskompetenzen 189-201.

${ }^{54}$ So die im Titel zum Ausdruck kommende Bewertung von HolensteIn, Stunde Null.

${ }^{55}$ Vgl. VATTER, Swiss Federalism 172. 
Begrenzung der Bundescompetenzen im Einzelnen ist augenfällig: und nicht mit Unrecht lässt sich der Verfassung vorwerfen, dass sie einem großen und kostbaren Palaste gleiche, in welchem wenig Räume wirklich benutzt werden" ${ }^{.56}$

\section{Bundesverfassung von 1874 Dammbruch zur Ausweitung der Bundeskompetenzen}

\section{a) Zunehmender Reformdruck und gescheiterte Reformen}

Das 19. Jahrhundert war von einem starken demographischen, ökonomischen und technischen Wandel geprägt, der nach 1860 den Ruf nach Reformen der staatlichen Strukturen lauter werden ließ. War die Schweiz um 1800 noch agrarisch geprägt, so wurde der Agrarsektor um 1870 vom Industriesektor bezüglich Wertschöpfung und Beschäftigtenzahl überholt. In die Zeit zwischen 1800 und 1900 wuchs zudem die Wohnbevölkerung um das doppelte. Bevölkerungswachstum und Industrialisierung verstärkten soziale Ungleichheiten, zeigten erste Auswirkungen auf die natürliche Umwelt und erhöhten das Bedürfnis nach staatlicher Steuerung der technischen Entwicklung, v.a. im Bereich des Eisenbahnwesens. Als politische Folge artikulierte sich seit den 1860er-Jahren eine demokratische Bewegung gegen die etablierten Kräfte des liberalen Bürgertums. Sie forderte eine verstärkte direktdemokratische Mitsprache, etwa durch die Mitwirkung des Stimmvolks an der Rechtsetzung über Referenden und Initiativen und die direkte Volkswahl von staatlichen Funktionsträgern. Die Diskussion um die päpstliche Unfehlbarkeit verschärfte sodann ab 1871 den Kulturkampf, der die schon bestehenden konfessionellen Gegensätze überlagerte und eine neue Konfliktlinie zwischen reformorientierten Katholi-

\footnotetext{
${ }^{56}$ BLUNTSCHLI, Geschichte 515
}

ken - etwa im josephinisch geprägten Fricktal und den „ultramontan“ orientierten konservativen Kräften aufbrechen ließ. Der deutsch-französische Krieg und die Deutsche Reichsgründung hinterließen zudem auch in der Schweiz Spuren. Der Krieg deckte Schwächen der Armee auf, die weitgehend aus kantonalen Kontingenten bestand. Die deutsche Einheit beflügelte diejenigen Kräfte, die sich mehr nationale Einheit wünschten, etwa durch Rechtsvereinheitlichung. ${ }^{57}$

Vor diesem Hintergrund entstand ein zunehmender Reformstau. Dieser führte dazu, dass die Bundesversammlung 1866 neun Vorlagen zur Teilrevision der Verfassung unterbreitete, die aber mit einer Ausnahme (Ausdehnung der Niederlassungsfreiheit auf Schweizerbürger jüdischen Glaubens) alle in der Abstimmung von Volk und Ständen abgelehnt wurden. 1872 folgte ein noch ambitionierteres Reformprojekt diesmal in Form einer umfassenden Totalrevision. Auch dieses Projekt scheiterte vergleichsweise knapp in der Abstimmung, wobei die französischsprachigen Kantone den Ausschlag gaben, die sich vor einer zu starken Zentralisierung und damit einer Dominanz der deutschsprachigen Mehrheit fürchteten. Die Vorlage wurde daraufhin mit Blick auf die Anliegen der französischsprachigen Schweiz weniger zentralistisch ausgestaltet. Zugleich wurden die kulturkämpferischen Bestimmungen noch verschärft. In der Abstimmung vom 19. April 1874 wurde die Totalrevision schließlich angenommen. ${ }^{58}$

\section{b) Grundsatz: Vollzugsföderalismus und Stärkung des Bundesgerichts}

Die Bundesverfassung von 1874 hielt - wie bereits erwähnt - am unveränderten Art. 3 fest. Vordergründig änderte sich damit am Grund-

\footnotetext{
${ }^{57}$ Hierzu KLEY, Bundesverfassung 31; SCHINDLER, Verwaltungsrecht, insb. Rz. 11.

${ }^{58}$ Vgl. KöLZ, Verfassungsgeschichte 599-626.
} 
muster der Kompetenzverteilung nichts. Dennoch hat die Totalrevision von 1874 einen eigentlichen Dammbruch ausgelöst und einen massiven "Peak“ der Kompetenzverlagerung auf den Bund bewirkt. ${ }^{59}$ Neben gesellschaftlichen und ökonomischen Faktoren dürfte hinter diesem Wandel auch das mit der Totalrevision zusammenhängende Bekenntnis zum Modell des Vollzugsföderalismus stehen. Die Verfassung von 1848 basierte noch auf der Grundannahme, dass die Bundeskompetenzen in erster Linie vom Bund und seinen Vollzugsorganen selbst wahrgenommen werden sollten - etwa durch Diplomaten des Bundes, eigene Post- und Zollbeamte oder eine Eidgenössische Münzstätte und die Pulvermühlen des Bundes. Indirekt kam dies darin zum Ausdruck, dass die BV 1848 den Bund dazu verpflichtete, bei der Rekrutierung seiner Beamten nach Möglichkeit die lokale Bevölkerung zu berücksichtigen (Art. 34 BV 1848). Hätte der Bund den Vollzug von Beginn weg den kantonalen und kommunalen Behörden belassen, wäre diese Vorgabe überflüssig gewesen. Vom Grundmodell des Bundesvollzugs verabschiedete sich der Bund nach 1874, indem er sich von nun an am Grundsatz orientierte: „Unifions, mais ne centralisons pas!“60 - Lasst uns (das Recht) vereinheitlichen, nicht aber (die Staatsgewalt) zentralisieren! ${ }^{61}$ Der Bund sollte sich also auf die Rechtsvereinheitli-

\footnotetext{
${ }^{59}$ DARDANELli/Mueller, De/Centralization, 149, 158.

${ }^{60}$ Zugeschrieben wird das Zitat dem radikal-liberalen, aber aus der zentralisierungsskeptischen lateinischen Schweiz stammenden Politiker Louis Ruchonnet (1834-1893); zit.n. His Staatsrecht 3, 426; vgl. auch FLEINER, Bundesstaatsrecht 24; KLEY, Verhältnis 626. Punktuell kam dieser Grundsatz auch im Text der Verfassung zum Ausdruck, so in Art. 20 Abs. 3 (Militärverwaltung, die ansonsten allerdings zentralisiert wurde) und Art. 64 Abs. 3 BV 1874 (Erwähnung der kantonalen Rechtsprechung). Später wurden diese ausdrücklichen Vorbehalte häufiger: SCHINDLER, Zusammenwirken 69f.

${ }^{61}$ Vgl. His, Bd. III, 425-427; SCHWEIZER, Staatsaufgaben 700 .
}

chung beschränken, den Vollzug aber den bestehenden Verwaltungs- und Justizbehörden der Kantone und Gemeinden überlassen. Auch wenn dieser Paradigmenwechsel erst mit der Reform von 1999 ausdrücklich Eingang in den Verfassungstext fand (vgl. Art. 46 BV 1999), wurde er bereits nach 1874 zur staatspolitischen Maxime bei der Begründung neuer Bundeskompetenzen. Damit wurde nicht nur föderalistischen Bedenken der lateinischen Schweiz Rechnung getragen, sondern auch dem erhöhten Bedürfnis nach demokratischer Legitimation und der in der Schweiz latent vorhanden Bürokratiefeindlichkeit. Die Funktionsträger der Kantone und Gemeinden wurden als Folge der demokratischen Bewegung vielerorts direkt vom Volk gewählt und übten ihr Amt oft als Neben- oder Ehrenamt im Rahmen ihrer staatsbürgerlichen Pflichten aus (sog. „Milizverwaltung“). Die am Berufsbeamtentum orientierte Bürokratie des Bundes erschien demgegenüber als „undemokratisch, nicht nur als Organisationsform, sondern auch dem ganzen in ihr herrschenden Geiste nach". ${ }^{62}$ Indem das Modell des Vollzugsföderalismus wegleitend wurde, konnte die politisch-emotionale Hemmschwelle zur Begründung neuer Bundeskompetenzen gesenkt werden. Im Alltag der Bürgerinnen und Bürger änderte sich durch eine Kompetenzverlagerung scheinbar nichts. Der Bund wurde vielmehr zur unsichtbar lenkenden Kraft im Hintergrund.

Mit der Totalrevision von 1874 wurde zudem das Bundesgericht institutionell gestärkt und seine Kompetenzen deutlich ausgeweitet (Art. 108-114 BV 1874). Das Bundesgericht sollte neu auch „Kompetenzkonflikte zwischen Bundesbehörden einerseits und Kantonalbehörden andererseits" beurteilen (Art. 113 Abs. 1 Z. 1 BV 1874). Das Bundesgericht konnte diese Aufgabe als "Hüterin der Kompetenzordnung" allerdings nur einseitig zu Gunsten des Bundes

\footnotetext{
${ }^{62}$ FLEINER, Bundesstaatsrecht 782.
} 
wahrnehmen, da die Bundesgesetze „für das Bundesgericht maßgebend" erklärt wurden (Art. 113 Abs. 3 BV 1874). Damit waren dem Gericht bei kompetenzwidrig erlassenen Gesetzen die Hände gebunden. Darüber hinaus entwickelte das Bundesgericht ein ausgesprochen unitarisches Selbstverständnis und verstand sich in erster Linie als "Hüterin der Rechtseinheit". ${ }^{63}$ Aus den Übergangsbestimmungen der Verfassung von 1874 (Art. 2) leitete das Gericht zudem $\mathrm{ab}$, dass dem Bundesrecht generell „derogatorische“ Kraft zukomme und der Grundsatz „Bundesrecht bricht kantonales Recht" als verfassungsmäßiges Individualrecht selbständig geltend gemacht werden könne. ${ }^{64}$ Die Bundesverfassung von 1874 und die Praxis des Bundesgerichts begründeten damit eine bis heute andauernde „hinkende Justiziabilität" der Kompetenzordnung. Während kompetenzwidriges Verhalten der Kantone gerichtlich sanktioniert werden kann, sind kompetenzwidrig erlassene Bundesgesetze weitgehend immunisiert.

\section{c) Konkrete Ausgestaltung}

\section{Von der Rechtseinheit zur Unübersichtlichkeit}

Die Totalrevision von 1874 stand unter dem Motto „Eine Armee, ein Recht". In diesem Sinn wurden die Zuständigkeiten des Bundes im Bereich der Armee ausgebaut (Art. 18-22 BV 1874) und der Bund erhielt die Kompetenz zur Vereinheitlichung des Zivilrechts, des Rechts des geistigen Eigentums sowie des Betreibungs- und Konkursrechts (Art. 64 BV 1874). Liberalen Interessen und der Stärkung des Binnenmarktes trugen sodann die Anerkennung der Handelsund Gewerbefreiheit (Art. 31 BV 1874) und die Möglichkeit der Vereinheitlichung von Berufsausweisen Rechnung (Art. 33 BV 1874). Umgekehrt wurden soziale Anliegen aufgenommen, indem der Bund beim Arbeitnehmerschutz und

\footnotetext{
${ }^{63}$ SCHINDLER, Rechtsanwendungsgleichheit 172.

${ }^{64}$ Kritisch BuRCKHARDT, Kommentar 823.
}

der Regulierung von Auswanderungsagenturen Zuständigkeiten erhielt (Art. 34 BV 1874). Verschiedene Bestimmungen der neuen Verfassung dienten sodann der Säkularisierung, etwa die Eindämmung kirchlicher Einflüsse im Zivilstands- und Begräbniswesen (Art. 53 BV 1874), im Eherecht (Art. 54 BV 1874) und die Abschaffung der geistlichen Gerichtsbarkeit (Art. 58 Abs. 2 BV 1874). Soziale, säkulare und demokratische Anliegen vereinigten sich beim Anspruch auf obligatorischen, kostenlosen und konfessionell neutralen Grundschulunterricht (Art. 27 Abs. 2-4 BV 1874). Sodann fand die technische Entwicklung und ihre Auswirkungen auf die natürliche Umwelt ihren Niederschlag in der Kompetenzordnung (Art. 26 BV 1874: Bundeskompetenz im Eisenbahnwesen; Art. 24 BV 1874: Wasserbau und Forstpolizei).

Während die Bundesverfassung von 1848 nur ein einziges Mal (1866) einer Teilrevision unterzogen wurde, war dies bei der Verfassung zwischen 1874 und 1999148 Mal der Fall.65 Die Dynamik der Verfassungsänderungen wurde beschleunigt durch die 1891 eingeführte Volksinitiative auf Partialrevision der Verfassung (Art. 118-123 BV). Die Partialrevisionen führten in zahlreichen Sachgebieten zu einer Ausweitung der Bundeskompetenzen. Sie können im Rahmen dieses Beitrags nicht vollständig und in all ihren Verästelungen wiedergegeben werden.66 Anders als im amerikanischen Verfassungsrecht wurden die Änderungen nicht als Anhänge hinzugefügt, sondern in den Verfassungstext integriert. Um die Nummerierung beibehalten zu können, wurden die Einschübe mit lateinischen Numeralien versehen, was der Übersichtlichkeit nicht eben zuträglich war. Im Zentrum dieser Teilrevisionen standen der sukzessive Ausbau des Sozialstaats seit Ende des 19. Jahrhunderts

\footnotetext{
65 SCHWEIZER, Staatsaufgaben 712.

66 Für eine empirisch-quantitative Analyse auf der Zeitachse vgl. insbesondere die Untersuchung von DARDANELLI/Mueller, De/Centralization, 138-160.
} 
und der Schutz strukturell schwacher Vertragsparteien im Arbeitsrecht, Mietrecht und Konsumentenrecht seit den 1970er-Jahren - hin zu einer eigentlichen „Sozialverfassung“ des Bundes. ${ }^{67}$ Sodann wurde die Kompetenz zur Rechtsvereinheitlichung 1898 vom Zivilrecht auf das Strafrecht ausgedehnt. ${ }^{68}$ Seit Beginn des 20. Jahrhunderts erfolgte ein Ausbau der Verkehrs- und Energieinfrastruktur („Infrastrukturverfassung“). ${ }^{69}$ Der Umweltschutz führte seit den 1950er-Jahren zu einer Reihe von zusätzlichen Bundeskompetenzen („Umweltverfassung“).70 In verschiedenen Phasen übernahm der Bund sodann eine zunehmend dominierende Rolle in der Wirtschafts- und Konjunkturpolitik („Wirtschaftsverfassung“). Dies galt insbesondere für den Ersten Weltkrieg, die Weltwirtschaftskrise und den Zweiten Weltkrieg. Viele der vom Bund ergriffenen Maßnahmen hatten ihre Grundlage vorerst in Dringlichkeitsbeschlüssen oder extrakonstitutionellem Notrecht (sog. „Vollmachtenregime“). Teilweise erhielten diese Maßnahmen erst nach dem Ende des Zweiten Weltkrieges eine ausdrückliche Grundlage in

\footnotetext{
${ }^{67}$ Kompetenz im Bereich Kranken- und Unfallversicherung (Art. 34 ${ }^{\text {bis }}$ von 1890), Alters- und Hinterlassenenversicherung (Art. 34quater und 41 ter von 1925), Brotgetreideversorgung (Art. 23 $3^{\text {bis }}$ von 1929), Wohnbauförderung (Art. 34 $4^{\text {sexies }}$ von 1972), Mieterschutz (Art. 34 ${ }^{\text {septies }}$ von 1972), berufliche Vorsorge (Art. 34quater $u$ d Art. $41^{\text {bis }}$ von 1972), Arbeitslosenversicherung (Art. 34 $4^{\text {novies }}$ von 1976), Konsumentenschutz (Art. 31 ies von 1981), Mieterschutz (Art. 34 $4^{\text {septies }}$ von 1986).

${ }^{68}$ Art. 64 ${ }^{\text {bis }}$ von 1898.

${ }^{69}$ Schifffahrt (Art. 24 ${ }^{\text {ter }}$ von 1919), Straßen- und Luftverkehr (Art. 37 bis und 37ter von 1921), Atomenergie und Strahlenschutz (Art. 24quinquies von 1957), Straßenbau (Art. 36 ${ }^{\text {bis }}$ von 1958), Rohrleitungsanlagen (Art. $26^{\text {bis }}$ von 1961).

${ }^{70}$ Gewässerschutz (Art. 24quater von 1953), Natur- und Heimatschutz (Art. 24 $4^{\text {sexies }}$ von 1962), Raumplanung (Art. 22quater von 1969), Umweltschutz (Art. 24 $4^{\text {septies }}$ 1971), Tierschutz (Art. 25 $5^{\text {bis }}$ von 1973), Moor- und Biotopschutz (Art. 24 ${ }^{\text {sexies }}$ Abs. 5 von 1987).
}

der Verfassung. ${ }^{71}$ Im Bereich der Wirtschaftspolitik verabschiedete sich der Bund sodann weitgehend vom Modell des Vollzugsföderalismus. Während der Bund schon seit 1848 über ein Münzregal verfügte, wurde sein Banknotenmonopol erst 1891 begründet, ${ }^{72}$ und 1905 wurde die Schweizerische Nationalbank ins Leben gerufen. In der Zwischenkriegszeit schuf der Bund die Preisbildungskommission (1927) als Vorläuferin der 1996 geschaffenen Wettbewerbskommission. 1934 erfolgte die Gründung der Bankenkommission, die 2009 in der Finanzmarktaufsichtsbehörde FINMA aufging. Besonders seit den 1990er-Jahren etablierten sich nach ausländischen Vorbildern weitere unabhängige Marktregulierungsbehörden des Bundes. ${ }^{73}$

\section{1960er-Jahre: „Helvetisches Malaise" und gescheiterte Reformversuche}

In den 1960er-Jahren wurde der Ruf nach einer erneuten Totalrevision der Bundesverfassung immer lauter. Die durch die geistige Landesverteidigung in den Kriegsjahren gefestigte „staatsbürgerliche Gesinnung" verlor ihre Selbstverständlichkeit. Und die sich abzeichnende Europäische Integration stellte vermeintlich Unverrückbares wie die Souveränität der Staaten in Frage. Zunehmend machte sich ein „Unbehagen im Kleinstaat" Schweiz breit. ${ }^{74}$ In seiner 1964 erschienenen Schrift „Helvetisches Malaise“ zeigte der Staatsrechtler und Politiker Max Imboden (1915-1969) den Reformstau in verschiedenen Bereichen des politischen Lebens auf und forderte einen "bewussten Neubau“ in Form einer

\footnotetext{
${ }^{71}$ Dies gilt insbesondere für die sog. Wirtschaftsartikel in Art. 31 ${ }^{\text {bis_-31quinquies }}$ von 1947.

72 Teilrevision von Art. 39 BV von 1874.

${ }^{73} \mathrm{Vgl}$. SCHINDLER, Verwaltungsrecht $329 \mathrm{f}$.

${ }^{74} \mathrm{Vgl}$. SCHMID, Unbehagen.
} 
Totalrevision der Bundesverfassung. ${ }^{75}$ Auch auf Druck des Parlaments wurden daher verschiedene Vorarbeiten für eine grundlegende Verfassungsreform in Angriff genommen. 1974 wurde schliesslich eine Expertengruppe unter dem damaligen Justizminister Kurt Furgler (1924-2008) eingesetzt, die 1977 einen Schlussbericht mit Verfassungsentwurf vorlegte. Dieser kam mit Blick auf die Kompetenzverteilung zwischen Bund und Kantonen zu einer wenig schmeichelhaften Einschätzung der geltenden Verfassung: „Der Bund erhielt eine Fülle neuer Bereiche zugewiesen - meist wirklich neue Aufgaben freilich und nicht solche, die im 19. Jahrhundert den Kantonen belassen werden sollten. Aber das Verhältnis der Kompetenzartikel zueinander wurde dunkel. Neue Bundesaufgaben traten unkoordiniert neben Grundrechts- und Organisationsbestimmungen. In den Bereichen, in denen der Bund nicht zur Gesetzgebung zuständig war, bildete die Zulässigkeit eidgenössischer Planung, Initiative, Finanzierung und Beratung ein Problem. Das verfassungsmässige System der Aufgabenverteilung wurde zudem immer mehr überwuchert durch Dringlichkeitsrecht. [...] Im Ergebnis sind daher heute die Kompetenzen von Bund und Kantonen in völlig unübersichtlicher Weise ineinander verfilzt und verflochten, ja man möchte geradezu sagen verkrallt."

Das Ziel der Experten war es, „dem Föderalismus die gebieterisch erforderliche neue Vitalität einzuflössen". ${ }^{77}$ Die neue Kompetenzordnung sollte sich daher an den Kriterien der „Schlichtheit, Klarheit und Flexibilität"78 orientieren. Die Schweiz wurde in Art. 1 des Entwurfs als „demokratischer, freiheitlicher und sozialer Bun-

\footnotetext{
75 IMBODEN, Helvetisches Malaise.

${ }^{76}$ Bericht der Expertenkommission für die Vorbereitung der Totalrevision einer Bundesverfassung (Bern 1977) 72.

77 Ebd. 10.

${ }^{78}$ Ebd. 94.
}

desstaat“ bezeichnet. Vorgeschlagen wurde, dass die Bundesverfassung keine präzis umschriebenen Kompetenzen mehr aufführen, sondern mit vergleichsweise offenen Formulierungen die „Verantwortungen" von Bund und Kantonen benennen sollte. In den Art. 50 und 51 wurden die „Hauptverantwortungen“ von Bund und (!) Kantonen aufgeführt. Die „übrigen Verantwortungsbereiche" nannte die Verfassung in Art. 52 hingegen nur beispielhaft. Hier galt - in Anlehnung an den bisherigen Art. 3 -, dass im Zweifel die Kantone verantwortlich gewesen wären. Allerdings hätte der Bundesgesetzgeber auch ohne Verfassungsänderung von dieser Grundregel abweichen können. Diese Neuerungen stießen v.a. bei den Kantonen auf Ablehnung. Sie „befürchteten einen Substanzverlust, wenn die Bundeskompetenzen nicht mehr abschliessend in der Verfassung aufgezählt würden" ${ }^{79}$ Nicht zuletzt am Widerstand der Kantone scheiterte dieses wohl $\mathrm{zu}$ ambitionierte Reformvorhaben einer grundlegenden Verfassungsrevision.

\section{Bundesverfassung von 1999 „Nachführung" im Zeichen der Kontinuität und Konsolidierung}

Am 12. Mai 1986 veröffentlichte der Staatsrechtler Kurt Eichenberger (1922-2005) in der Neuen Zürcher Zeitung einen Beitrag mit dem Titel „Realitätsgebundene Verfassungsrevision“ und popagierte das Modell einer "Nachführung“. Diese sollte in erster Linie darauf gerichtet sein, Verfassungsurkunde und Verfassungspraxis wieder ein Einklang zu bringen, die Verfassung sprachlich verständlich aufzubereiten und systematisch übersichtlich $\mathrm{zu}$ ordnen. Diesem Nachführungsgedanken war die letzte Totalrevision der Bundesverfassung verpflichtet, die

\footnotetext{
${ }^{79}$ Botschaft über eine neue Bundesverfassung vom 20. 11. 1996, BBl. 1997 I 1, 27.
} 
am 18. April 1999 von Volk und Ständen angenommen wurde.

Die Bundesverfassung von 1999 ist somit - gerade mit Blick auf den Föderalismus - stark vom Bemühen um Kontinuität geprägt. ${ }^{80}$ Der Begriff „Bundesstaat" wird weiterhin vermieden. Die Kantone werden im Art. 1 in der tradierten Reihenfolge (und nicht alphabetisch) aufgeführt. Und die Schlüsselnorm zur Kompetenzverteilung - Art. 3 BV - bleibt abgesehen von einer sprachlichen Glättung unangetastet. Das Verhältnis zwischen Bund und Kantonen wird nun allerdings systematisch und übersichtlich in einem eigenen Titel der Bundesverfassung geordnet. Ein erster Abschnitt zum „Verhältnis von Bund und Kantonen“ versucht, die Grundsätze des gelebten Föderalismus im Sinne von eher programmatischen Normen zu skizzieren. Mit Blick auf die Aufgabenteilung ist v.a. das ausdrückliche Bekenntnis zum Vollzugsföderalismus in Art. 46 zu nennen. In einem zweiten Abschnitt werden die Zuständigkeiten des Bundes geordnet und thematisch gegliedert, was gegenüber der Vorgängerverfassung die Orientierung stark erleichtert. Auch wenn die BV in erster Linie die Zuständigkeiten des Bundes aufführt, nennt sie punktuell auch Kompetenzen der Kantone: Sei dies, um (deklaratorisch) die Abgrenzung zur Bundeszuständigkeit zu klären (etwa in Art. 62 Abs. 1 BV: Zuständigkeit der Kantone für das Schulwesen), oder im Sinne eines echten Vorbehalts, um den Kantonen eine Kompetenz einzuräumen, die in eine umfassende Bundeszuständigkeit fällt (etwa Art. 56 BV: Beziehungen der Kantone zum Ausland - trotz umfassender Zuständigkeit des Bundes im Bereich der Außenpolitik: Art. 54 BV). ${ }^{81}$

Auch wenn die Totalrevision von 1999 in erster Linie vom Modell einer bewahrenden „Nachführung" geprägt war, sollte sie doch Basis für

\footnotetext{
${ }^{80}$ Eingehend RHINOW, Bundesstaatsreform 63-92.

${ }^{81} \mathrm{Vgl}$. EhrenZELLER, EHRENZELLER, Kompetenzzuteilung 38f.
}

mehrere Reformvorhaben bilden, die jedoch in getrennten und zeitlich gestaffelten Vorlagen Volk und Ständen zur Abstimmung unterbreitet wurden. Zwei davon wirkten sich auch auf das Verhältnis zwischen Bund und Kantonen aus. In konsequenter Fortführung der bereits 1874 verfolgten Reformbemühungen sollte mit der „Justizreform" die Rechtseinheit und der Rechtsschutz gestärkt werden. Diese Reform wurde am 12. März 2000 angenommen und begründete eine neue Bundeszuständigkeit zur Vereinheitlichung des Zivil- und Strafprozessrechts (Art. 122 Abs. 1 und Art. 122 Abs. 1 BV). Am 1. Januar 2011 traten die Zivilprozessordnung und die Strafprozessordnung in Kraft, womit nun das ganze materielle und formelle Zivilund Strafrecht schweizweit einheitlich geregelt sind. Die mit der Justizreform eingeführte Rechtsweggarantie (Art. 29a BV) verpflichtete die Kantone dazu, die noch bestehenden Lücken im verwaltungsgerichtlichen Rechtsschutz zu schließen.

Neben der "Justizreform" wurde am 28. November 2004 eine „Föderalismusreform“ angenommen, deren Hauptfokus aber weniger auf der Zuständigkeitsordnung als auf dem Finanzausgleich lag. Allerdings wurde die Verfassung um Grundsatzbestimmungen angereichert, die auf programmatische Weise die Idee der Subsidiarität bei der Zuweisung von Staatsaufgaben unterstreichen sollen (Art. 5a und Art.43a BV). Diese „schon fast ins Inflationäre gehende $\mathrm{Zu}$ nahme föderalistischer Grundsatzbekenntnisse, selbstverständlicher bundesstaatlicher Maximen und substanzarmer ökonomischer Lehrsätze" ${ }^{\text {"82 }}$ wirkt etwas befremdend vor dem Hintergrund, dass gleichzeitig in Art. 48a BV die Möglichkeit geschaffen wurde, interkantonale Verträge für alle Kantone „allgemein verbindlich“ zu erklären oder einzelne Kantone zur Beteiligung an

\footnotetext{
82 BIAGGINI, Kommentar, Vorbemerkungen zu Art. 42135, Rz. 5.
} 
Verträgen zu verpflichten. Diese Möglichkeit zur Zwangskooperation jenseits von Bundeszuständigkeiten wurde in der rechtswissenschaftlichen Literatur fast einhellig kritisiert und hat in der Staatspraxis bislang keine Wirkung entfaltet. ${ }^{83}$ Diese eigenartige Diskrepanz zwischen föderalistischem Lippenbekenntnis und tatsächlicher Stärkung der Bundesgewalt erinnert in gewisser Weise an die staatenbündische Rhetorik der Bundesverfassung von 1848.

\section{Gegenwart}

\section{Vollzugskompetenz als „ultimum refugium kantonaler Staatlichkeit"?}

Die Kompetenzverteilung zwischen Bund und Kantonen ist in der Schweiz einerseits von großer Kontinuität geprägt. Dies kommt exemplarisch im unveränderten Art. 3 aller bisherigen Bundesverfassungen zum Ausdruck, der wie ein unverrückbarer Eckpfeiler der föderalen Kompetenzordnung erscheint. Im Bereich der Staatsgewalt und Außenpolitik verfestigte sich sodann eine Aufgabenteilung zwischen Bund und Kantonen, die ihre Ursprünge bereits in der Zeit vor 1798 hatte. Der Bund nimmt die gemeinsamen Interessen gegen "Außen“ war, er sichert die Grenzen und ist für die Landesverteidigung zuständig. Der Bund erscheint aus völkerrechtlicher Sicht der Träger der staatlichen Souveränität. Im „Innern“ hingegen verteidigen die Kantone ihre Polizeihoheit und das Gewaltmonopol bis heute erfolgreich. Alle Versuche, eine bewaffnete Sicherheits- oder Vollzugspolizei des Bundes zu schaffen, sind bislang gescheitert. Gegenüber den Individuen tritt die hoheitliche Staatsgewalt daher bis heute vor allem in Gestalt der kantonalen und kommunalen Polizeikorps und Hoheitsverwaltung in Erscheinung. Die

\footnotetext{
${ }^{83}$ Stellvertretend aus der Fülle der Literatur BIAGGINI, Kommentar, Vorbemerkungen zu Art. 42-135, Rz. 5; RHINOW, Bundesstaatsreform 73-91.
}

Staatsgewalt des Bundes ist im Alltag heute sogar weniger sichtbar als noch im späten 19. und im 20. Jahrhundert. Die ehemals uniformierten Post- und Bahnbeamten wurden durch Mitarbeitende von öffentlichen Unternehmen abgelöst, die jeden obrigkeitlichen Charakter zu vermeiden suchen. Die Grenzkontrollen durch das Grenzwachtkorps des Bundes wurden seit dem Beitritt der Schweiz zum Schengen-Raum (2009) stark ausgedünnt und auch die Armee hat ihre Bedeutung als schweizweit identitätsbildende Klammer eingebüßt.

Kantone und Gemeinden stellen vor diesem Hintergrund bis heute die Basis des schweizerischen Staatswesens dar. Hierauf deutet aus empirischer Sicht auch ihr sehr hoher Anteil an den Staatsausgaben hin. ${ }^{84}$ Und der Anteil des Bundespersonals am gesamten Staatspersonal ist mit weniger als 10\% im internationalen Quervergleich $\mathrm{zu}$ anderen Bundesstaaten sehr tief. ${ }^{85}$ Nicht geleugnet werden kann aber, dass seit 1874 die Bundeskompetenzen massiv angewachsen sind. Auch wenn der Bund im Verwaltungsalltag heute weniger sichtbar ist als früher, erscheint er doch als die bestimmende Kraft im Hintergrund, die zahlreiche Fäden lenkend in der Hand hält. Zentrale Bereiche der Staatstätigkeit und des Alltagslebens werden heute von bundesrechtlichen Normen bestimmt. ${ }^{86}$ Hinzu kommt die kontinuierlich wachsende Bedeutung staatsvertragsrechtlicher Normen. Hier versagt die in der Verfassung verankerte Kompetenzzuweisung, da der Bund für die Auswär-

\footnotetext{
${ }^{84}$ LADNER, Föderalismus, 125f.: Während der Anteil des Bundes an den Staatsausgaben 1850 bei nur 7\% lag, stieg er nach Ende des Zweiten Weltkriegs auf $37,7 \%$ (1950) und hat sich seit den 1970er-Jahren bei ca. einem Drittel eingependelt. Vgl. auch VATTER, Swiss Federalism, 181.

${ }^{85}$ LADNER, Föderalismus 139-141.

$86 \mathrm{Vgl}$. die eingehende Analyse nach verschiedenen Politikbereichen DARDANELLI/MuelLER, De/Centralization 146-160. Vgl. auch VATTER, Swiss Federalism 169-183.
} 
tigen Angelegenheiten umfassend zuständig ist (Art. $54 \mathrm{BV}$ ) - somit auch dort, wo gemäß Bundesverfassung eine kantonale Zuständigkeit vorliegt. Oder um die Metapher Bluntschlis aufzugreifen: Inzwischen werden nicht nur die Säle des Bundespalastes intensiv genutzt; Bundesrecht und internationales Recht bestimmen heute auch das Leben in den Räumen der Kantone und den Amtsstuben der Gemeinden. Wenn wir uns als konkretes Beispiel zum Schluss noch einmal den Gerichtssaal in Laufenburg ansehen, dann stehen dort inzwischen nur noch Gesetze des Bundes:87 Bis 1826 wurde noch nach dem Recht geurteilt, wie es unter Habsburgischer Herrschaft galt. Nach 1826 erfolgte die sukzessive Kodifikation des Zivilrechts durch das von österreichischen Vorlagen geprägte Allgemeine Bürgerliche Gesetzbuch für den Kanton Aargau, die erst 1847 abgeschlossen wurde. 1883 wurde das kantonale Recht mit dem Obligationenrecht erstmals durch bundesrechtliche Vorschriften verdrängt, und seit 1912 war mit dem Zivilgesetzbuch das gesamte Privatrecht bundesrechtlich kodifiziert. 1942 trat schliesslich ein einheitliches Strafgesetzbuch des Bundes in Kraft, und seit 2011 wenden die Richterinnen und Richter in Laufenburg auch die gesamtschweizerischen Zivil- und Strafprozessordnungen an. Trotz dieser Dominanz des Bundesrechts bleibt das Gericht aber ein Bezirksgericht des Kantons Aargau und die Richterinnen und Richter werden alle vier Jahre von den Stimmberechtigten des Bezirks Laufenburg gewählt.

Vor diesem Hintergrund zeichnet sich zu Beginn des 21. Jahrhunderts immer deutlicher eine funktionale Ausdifferenzierung der föderalen Zuständigkeitsordnung ab. Art. 3 BV mag seine

\footnotetext{
${ }^{87}$ Bezirksgerichte sind als erstinstanzliche Gerichte der „ordentlichen“ Gerichtsbarkeit in aller Regel nicht für verwaltungsrechtliche Streitigkeiten zuständig. Vor den kantonalen Verwaltungsgerichten findet neben Bundesrecht auch kantonales Recht und insb. das kantonale Verfahrensrecht Anwendung.
}

Ursprünge im amerikanischen Verfassungsrecht haben, spätestens seit 1874 hat sich der schweizerische Föderalismus aber in eine andere Richtung entwickelt. Während sich in den USA zwei voll ausgebaute Staatsebenen entwickelt haben, erscheinen Bund und Kantone sehr viel stärker ineinander verschränkt. Man kann diese beiden Ausprägungen auch als "layer cake-“ und "marble cake-federalism" bezeichnen. Während die Föderative (Außenpolitik und Verteidigung) schon in der Alten Eidgenossenschaft weitgehend als gemeinsame Aufgabe betrachtet wurde, verlagerte sich im Verlauf des 20. Jahrhunderts auch im Bereich der Legislative und Gubernative der Schwerpunkt auf den Bund. Demgegenüber erscheinen der Vollzug durch Administrative und Judikative bis heute als „ultimum refugium kantonaler Staatlichkeit" ${ }^{\prime} 88$ Aus einer rein rechtlichen Perspektive mag dies als massiver Bedeutungsverlust kantonaler Eigenstaatlichkeit oder als "Zentralisierung“ erscheinen. Nicht übersehen werden darf aber, dass die Kantone im Rahmen der Umsetzung von Bundesrecht über erhebliche Spielräume verfügen und diese auch nutzen. Der Bund macht von den ihm zur Verfügung stehenden Aufsichtsmitteln nur sehr zurückhaltend Gebrauch. ${ }^{89}$ Die Kantone wiederum gehören zu den wichtigsten Akteuren der Bundespolitik und üben einen nicht zu unterschätzenden Einfluss auf die Gesetzgebung des Bundes aus. ${ }^{90}$ Ohne Kantone (und Gemeinden) ist das schweizerische Staatswesen bis heute kaum vorstellbar - auch wenn sich die Zuständigkeiten innerhalb der föderalen Strukturen der Eidgenossenschaft in den letzten 300 Jahren grundlegend gewandelt haben.

\footnotetext{
${ }^{88}$ SCHINDLER, Zusammenwirken 70; EHRENZELLER, EHRENZELLER, Kompetenzzuteilung 65. Vgl. VATTER, Swiss Federalism, 182, der von „administrative federalism" spricht.

${ }^{89}$ SCHINDLER, Rechtsanwendungsgleichheit 174-184.

${ }^{90}$ LADNER, Föderalismus 52-55.
} 


\section{Korrespondenz:}

Prof. Dr. Benjamin SCHINDLER

Universität St. Gallen

Lehrstuhl für öffentliches Recht

Tigerbergstraße 21

CH-9000 St. Gallen

benjamin.schindler@unisg.ch

ORCID-Nr. 0000-0001-5352-5714

\section{Abkürzungen:}

ASHR Amtliche Sammlung der Acten aus der Zeit der Helvetischen Republik

BBl. Bundesblatt

BV 1815 Bundesvertrag 1815

BV Bundesverfassung

Siehe auch das allgemeine Abkürzungsverzeichnis: [http://www.rechtsgeschichte.at/media/abk.pdf]

\section{Literatur:}

Amtliche Sammlung der Acten aus der Zeit der Helvetischen Republik (ASHR), Bd.1 (Bern 1886), Bd. 6 (Bern 1897).

Giovanni BIAGGINI, Bundesverfassung der Schweizerischen Eidgenossenschaft, Kommentar (Zürich 22017).

Johann Jakob Blumer, Joseph Karl Pankraz MoReL, Handbuch des Schweizerischen Bundesstaatsrechtes, Bd. 1 (Basel ${ }^{3}$ 1891).

Johann Caspar BLUNTSCHLI, Geschichte des schweizerischen Bundesrechtes, Bd. 1 (Stuttgart ${ }^{21875) .}$

Franz Michael BÜELER, Compendium des gemeinen eidgenössischen Rechts (1697), in: Zeitschrift für Schweizerisches Recht 16 (1869) 45-154.

Walther BURCKHARDT, Kommentar der schweizerischen Bundesverfassung vom 29. Mai 1874 (Bern $\left.{ }^{3} 1931\right)$.

Paolo Dardanelli, Sean Mueller, Dynamic De/Centralization in Switzerland, 1848-2010, in: Publius, The Journal of Federalism 49/2019, 138-165.

Jakob DuBS, Das Oeffentliche Recht der Schweizerischen Eidgenossenschaft, Theil 2 (Zürich 1878).

Bernhard EHRENZELLER, Kaspar EHRENZELLER, "L'amour de la complexité" im Bundesstaat Schweiz, Kompetenzzuteilung und Kompetenzwahrnehmung in Bund und Kantonen, in: Anna GAMPER u.a. (Hgg.), Föderale Kompetenzverteilung in Europa (Baden-Baden 2016) 33-66.
Fritz FLEINER, Schweizerisches Bundesstaatsrecht (Tübingen 1923).

DERS., Zaccaria GIACOMETTI, Schweizerisches Bundesstaatsrecht (Zürich 1949).

Ulrich HÄFELIN u.a., Schweizerisches Bundesstaatsrecht (Zürich-Basel-Genf ${ }^{102020)}$.

Eduard HIS, Geschichte des neuern Schweizerischen Staatsrechts, Bd. 1 (Basel 1920), Bd. 3 (Basel 1938).

Rolf HolEnsteIN, Stunde Null, Die Neuerfindung der Schweiz 1848, Die Privatprotokolle und Geheimberichte (Basel 2018).

Max IMBODEN, Helvetisches Malaise (Zürich 1964).

Isaak IsELIN, Tentamen iuris publici Helvetici (1751), in: Schriften zur Politik, hg. von Florian GELzER (Basel 2014).

Jakob KAISER (Hg.), Amtliche Sammlung der neuern Eidgenössischen Abschiede (Bern 1876).

Andreas KLEY, Bundeskompetenzen mit ursprünglich derogatorischer Wirkung aus historischer Perspektive, in: recht 17 (1999) 189-201.

DERS., Bundesverfassung, in: Marco JORIO (Hg.), Historisches Lexikon der Schweiz, Bd. 3 (Basel 2004) 27-35.

DERS., Das Verhältnis von Föderalismus und Demokratie, in: Oliver Diggelmann, Maya Hertig RANDALL, Benjamin SCHINDLER (Hgg.), Verfassungsrecht der Schweiz, Bd. 1 (Zürich-Basel-Genf 2020) 621-637.

Alfred KÖLZ, Neuere schweizerische Verfassungsgeschichte, ihre Grundlinien in Bund und Kantonen seit 1848 (Bern 2004).

Andreas LADNER, Der Schweizer Föderalismus im Wandel, Überlegungen und empirische Befunde zur territorialen Gliederung und der Organisation der staatlichen Aufgabenerbringung in der Schweiz (Lausanne 2018).

John LOCKE, Two Treatises of Government (London 1690).

Thomas MAISSEN, Die Geburt der Republic, Staatsverständnis und Repräsentation in der frühneuzeitlichen Eidgenossenschaft (Göttingen 2006).

Victor MONNIER, Alfred KÖLZ, Bonaparte et la Suisse: travaux préparatoires de l'Acte de Médiation (1803): procès-verbal des assemblées générales des députés helvétiques et des opérations de la Commission nommée par le Premier Consul pour conférer avec eux (Genf 2002).

René RHINOW, Bundesstaatsreform und Demokratie. Der schweizerische Föderalismus aus rechtlicher Sicht, in: René L. FreY (Hg.), Föderalismus - zukunftstauglich?! (Zürich 2005) 63-92.

Ders., Markus SchefER, Peter UebersAX, Schweizerisches Verfassungsrecht (Basel ${ }^{32016)}$ 63-92. 
Johann Jakob RÜTTIMANN, Das nordamerikanische Bundesstaatsrecht verglichen mit den politischen Einrichtungen der Schweiz, Theil 1 (Zürich 1867).

Benjamin SCHINDLER, Grundlagen des Verwaltungsrechts - Staat, Verwaltung und Verwaltungsrecht: Schweiz (§ 49), in: Armin von BogDANDY, Sabino CAssese, Peter M. Huber (Hgg.), Handbuch Ius Publicum Europaeum, Bd.3, Verwaltungsrecht (Heidelberg 2010) 311-347.

DERS., Rechtsanwendungsgleichheit in Mehrebenensystemen (= Veröffentlichungen der Vereinigung der Deutschen Staatsrechtslehrer 77, Heidelberg 2019) 167-209.

Dietrich SCHINDLER (jun.), Das Zusammenwirken zwischen Bundesverwaltung und kantonalen Verwaltungen, in: Jahrbuch der Schweizerischen Vereinigung für politische Wissenschaften 4 (1964) 61-82.

Karl SCHMID, Unbehagen im Kleinstaat (Zürich-München 1963).

Jakob SCHOLLENBERGER, Bundesverfassung der Schweizerischen Eidgenossenschaft, Kommentar mit Einleitung (Berlin 1905).
Rainer J. SCHWEIZER, Verteilung der Staatsaufgaben zwischen Bund und Kantonen, in: Oliver DigGELMANN, Maya Hertig RANDALl, Benjamin SCHINDLER (Hgg.), Verfassungsrecht der Schweiz, Bd. 1 (Zürich-Basel-Genf 2020) 691-713.

Josias SIMLER, Von dem Regiment der Loblichen Eydgenossenschaft (Zürich 1722).

Alexis De Tocqueville, De la démocratie en Amérique (Paris ${ }^{12} 1848$ ).

Pierre TsCHANNEN, Staatsrecht der Schweizerischen Eidgenossenschaft (Bern ${ }^{52021)}$.

Adrian VATTER, Swiss Federalism, The Transformation of a Federal Model (London-New York 2018).

Andreas WÜRGLER, Geschichte des schweizerischen Verfassungsrechts bis 1798, in: Oliver DigGELMANN, Maya HeRTIG RANDALL, Benjamin SCHINDLER (Hgg.), Verfassungsrecht der Schweiz, Bd. 1 (Zürich-Basel-Genf 2020) 31-56.

DERS., Die Tagsatzung der Eidgenossen, Politik, Kommunikation und Symbolik einer repräsentativen Institution im europäischen Kontext (1470-1798) (Epfendorf/Neckar 2013). 